\title{
Need for cognitive closure as determinant for guidance in wiki-based learning
}

\section{Sven Heimbuch ${ }^{1}$ and Daniel Bodemer ${ }^{1}$}

\author{
${ }^{1}$ University of Duisburg-Essen, Germany \\ Corresponding author: Sven Heimbuch \\ Email address: sven.heimbuch@uni-due.de
}

\begin{abstract}
In wikis as collaborative knowledge construction environments for learning the outcome and its underlying processes can be considered on an individual as well as on a social system's level. In previous research, we could show that by implementing either supplemental implicit or explicit guidance focused on wiki discussions positive effects on the learner's side could be achieved. This study investigates what type of guidance implemented on the level of talk page discussions is beneficial dependent on the learner's degree of need for cognitive closure and if an interaction of these both factors produces larger positive effects on the individual learning processes and the resulting outcome. Therefore, we are conducting a 2 $x 2$ between-subjects design experimental study comparing four groups contrasting high vs low need for cognitive closure participants and implicit vs explicit guidance measures that we have positively evaluated in previous work. We expect to gather evidence that fostering learning processes in wiki-based settings making use of talk page discussions is highly dependent on individual cognitive variables interacting with the type of provided additional guidance.
\end{abstract}

\section{INTRODUCTION}

In computer-supported collaborative learning there is an ever-growing number of research covering supplemental web-based learning environments, such as wikis, to facilitate knowledge construction processes within the individual learner and the social system itself. In previous work on the co-evolution of knowledge in social system, Cress and Kimmerle (2008) discussed the occurrence of internalisation and externalisation processes of knowledge artefacts into an individual cognitive system as well as into the wiki as social system, which are similar and analogous to those processes originally discussed in Piaget's theories of equilibration. The mutual influences of either system on the other open up prospects for the emergence of socio-cognitive conflicts through possible dissents between an individual's and the social system's knowledge base.

Such conflicts that can arise from information contradicting the other system's knowledge base do not have to be detrimental for learning and making use of its beneficial potentials plays an important role in collaborative learning scenarios (Mugny and Doise, 1978). The induction and confrontation with conflicts that are grounded on different perspectives or contradictory facts can trigger reorganisation and restructuring of cognitive structures. Resulting alteration processes of an individual's cognitive representation of knowledge about specific contents are strengthened while trying to reach a consensus or feeling the need for a common understanding (Bell et al., 1985).

Supportive measures for dealing with sociocognitive conflicts in web-based learning environments that have proven to be effective for participants in different contexts range from deployments of implicit guidance approaches, e.g. implementation of cognitive group awareness representations (Janssen and Bodemer, 2013), to more explicit instructional methods, i.e. instructional designs through collaboration scripts (Dillenbourg, 2002). Wiki talk pages comprise hidden potentials for collaborative knowledge construction purposes that should be made more salient to interested users and 
learners by providing them additional guidance on the level of discussion threads. Visual feedbacks as external representations of group awareness information have been realised as multidimensional graphs or highlighting emphases of specific aspects of interest. Such visualisations can be helpful cues for readers of large online forum discussions that can also be found on wiki talk pages to navigate through the contents and select the most relevant information, e.g. the occurrence of content-related controversies (Heimbuch and Bodemer, 2014). The deployment of such cognitive group awareness representations that gather and visualise knowledgerelated information have been successfully implemented as implicit guidance measures to structure learning processes in wiki-based environments (Heimbuch and Bodemer, 2015).

Research on collaboration scripts has also been gathering evidence that explicit instructions to groups can be effective to achieve significant learning effects in wiki-related research. Instructions aiming at the improvement of collaborative revision processes that set the focus on increased coordination prior to any integration of knowledge artefacts can lead to less redundant revisions and more coherent texts (Wichmann and Rummel, 2013). Similar wiki-related collaboration scripts with regard to a more intensive a priori exchange of different points of view and opposing arguments facilitated learners to acquire contrary pieces of information and integrate these into their individual cognitive systems, which resulted in more elaborated responses to a historically controversial topic (Heimbuch and Bodemer, 2015).

Regardless of the type of deployed supportive measure for individual learners focussing on sociocognitive conflicts in collaborative settings such as wikis, the consideration of differences in specific personality and cognitive differences plays an important role. In settings as the aforementioned, research has identified the personal Need for Cognitive Closure as a relevant construct when learners are confronted with conflicts and controversies induced by ambiguous or contradictory information (Webster and Kruglanski, 1994). A person with a high need for cognitive closure tends to avoid ambiguity and searches for plausible but quick solution to a problem. In contrast to that, low need for cognitive closure individuals show preferences towards ambiguity and mostly enjoy participating in discussions and more extensive information search.

Recent research on wiki-based learning scenarios with implementations of implicit and explicit measures of supporting learners could confirm the influences of the individual need for closure on the learning outcome and the underlying processes (Heimbuch and Bodemer, 2015). For this current study we are building upon the results of our previous work where the need for cognitive closure has been identified as influential variable on learning in wiki-based environments. Therefore, we are mainly interested in the question if we are able to identify a significant interaction between the degree of an individual's need for cognitive closure and the type of provided guidance (implicit vs explicit) that have already been deployed and their positive outcomes have been confirmed in previous studies.

\section{METHODS}

To answer our main research question of interest, an experimental study is currently conducted in a controlled laboratory setting. We are aiming at researching approximately $N=180$ students in a balanced two factorial between-subjects design. Figure 1 illustrates the setups of the wiki environments for the experimental groups that correspond to the study's first independent factor Guidance, where (1) visual representations of a discussion's controversy occurrence and its status are implemented as implicit guidance and (2) a collaboration script with a focus on discussing changes to the wiki prior to editing is applied as explicit guidance for learners. For the second factor, we are conducting a pre-study on students' level of Need for Cognitive Closure (NCC) and categorise them into high and low closure.

Independent of the experiment's guidance type, the overarching task for all groups is to edit an original article relying on new information and evidence found inside the discussion threads and to participate in a number of talks. The article and discussion 


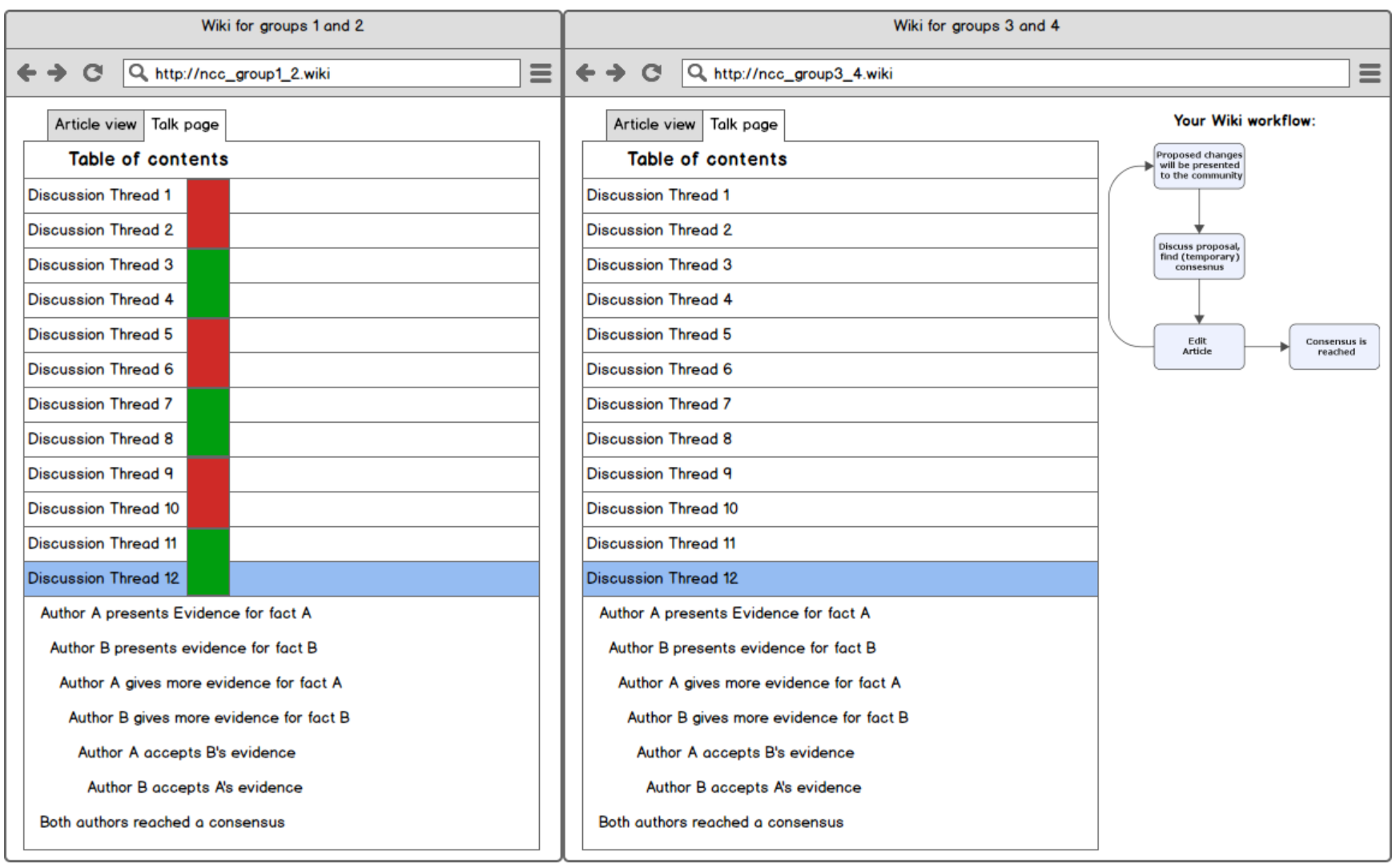

Figure 1. Mockup illustrations of the wikis for implicitly guided (controversy occurrence plus resolution status) groups 1 and 2 (left) and explicitly guided "discus" collaboration script) groups 3 and 4 (right).

contents of this study are on a number of different topics covering renewable and fossil energy sources for which contradictory information and opposing points of view on several aspects exist. The currently conducted study is scheduled to be finished until mid-November.

\section{OUTLOOK}

To measure the outcome with regard to learning success we will process and evaluate the answers given in a knowledge test about the study's contents. With regard to underlying processes we plan to analyse recorded log data on clicks and wiki activities, such as reading and writing times at each of the study's phases. Furthermore, a number of qualitative analyses on the contents of the edited texts of a random selection from the four experimental groups will be conducted. At all analytical stages we will analyse the effects of the main interested influencing variable Need for Cognitive Closure as well as the effects from other potentially relevant influencing variables.

\section{REFERENCES}

Bell, N., Grossen, M., and Perret-Clermont, A.-N. (1985). Sociocognitive conflict and intellectual growth. New Directions for Child and Adolescent Development, 1985(29):41-54.

Cress, U. and Kimmerle, J. (2008). A systemic and cognitive view on collaborative knowledge building with wikis. International Journal of Computer-Supported Collaborative Learning, 3(2):105-122.

Dillenbourg, P. (2002). Over-scripting CSCL: The risks of blending collaborative learning with instructional design. Three worlds of CSCL. Can we support CSCL?, pages 61-91.

Heimbuch, S. and Bodemer, D. (2014). Supporting awareness of content-related controversies in a wiki-based learning environment. In Proceedings 
of The International Symposium on Open Collaboration, pages 30:1-4, New York, NY, USA. ACM.

Heimbuch, S. and Bodemer, D. (2015). Let's Talk about Talks: Supporting Knowledge Exchange Processes on Wiki Discussion Pages. In Ninth International AAAI Conference on Weblogs and Social Media (ICWSM-15), pages 56-61. AAAI Press.

Janssen, J. and Bodemer, D. (2013). Coordinated computer-supported collaborative learning: Awareness and awareness tools. Educational Psychologist, 48(1):40-55.

Mugny, G. and Doise, W. (1978). Socio-cognitive conflict and structure of individual and collective performances. European Journal of Social Psychology, 8(2):181-192.

Webster, D. M. and Kruglanski, A. W. (1994). Individual differences in need for cognitive closure. Journal of Personality and Social Psychology, 67(6):1049-1062.

Wichmann, A. and Rummel, N. (2013). Improving revision in wiki-based writing: Coordination pays off. Computers \& Education, 62:262-270. 